OPEN ACCESS

Edited by:

Oliver Kepp,

Institut National de la Santé et de la

Recherche Médicale

(INSERM), France

Reviewed by:

Abhijit Nair,

Ministry of Health, Oman

*Correspondence:

Julio Montejano

Julio.montejano@cuanschtuz.edu

Specialty section:

This article was submitted to

Surgical Oncology,

a section of the journal

Frontiers in Oncology

Received: 27 October 2021

Accepted: 08 December 2021

Published: 23 December 2021

Citation:

Montejano $J$ and Jevtovic-Todorovic $V$ (2021) Anesthesia and Cancer, Friend

or Foe? A Narrative Review.

Front. Oncol. 11:803266.

doi: 10.3389/fonc. 2021.803266

\section{Anesthesia and Cancer, Friend or Foe? A Narrative Review}

\author{
Julio Montejano * and Vesna Jevtovic-Todorovic \\ School of Medicine, University of Colorado, Aurora, CO, United States
}

Cancer remains the leading cause of death worldwide with close to 10 million deaths reported annually. Due to growth of the advanced age cohort in our population, it is predicted that the number of new cancer cases diagnosed between now until 2035 is to reach potentially 24 million individuals, a staggering increase in a relatively short time period. For many solid tumors, surgical resection along with chemotherapy is the best available approach to a potential cure which leads to almost $80 \%$ of cancer patients undergoing at least one surgical procedure during the course of their disease. During surgical intervention, the exposure to general anesthesia can be lengthy, complex and often involves various modalities resulting in an important question as to the role, if any, anesthesia may play in primary recurrence or metastatic conversion. Many components of the stress and inflammatory responses exhibited in the perioperative period can contribute to cancer growth and invasion. The agents used to induce and maintain general anesthesia have variable interactions with the immune and neuroendocrine systems and can influence the stress response during surgery. Thus, debating the best type of anesthesia that would help to attenuate sympathetic and/or pro-inflammatory responses while modulating cytokine release and transcription factors/oncogenes remains at the forefront. This may affect inducible cancer cell survival and migratory abilities not only intra-operatively, but also during the immediate post-operative phase of recovery. The ultimate question becomes how and whether the choice of anesthesia may influence the outcomes of cancer surgery with two major approaches being considered, i.e., regional and general anesthesia as well as the various hypnotics, analgesics and sympatholytics commonly used. In this review, we will address the latest information as to the role that anesthesia may play during cancer surgery with specific focus on primary recurrence and metastasis.

Keywords: cancer recurrence, metastatic conversion, general anesthesia, regional anesthesia, total intravenous anesthesia, dexmedetomidine, lidocaine infusion

\section{INTRODUCTION}

Although more is known about cancer biology and treatment today than ever before, cancer remains the leading cause of death worldwide and it is predicted that this death toll will only continue to increase owed to our ever-aging population (1-3). The perioperative period presents a unique conundrum for the perioperative care team; patients present for surgery to be cured of their disease and yet find themselves 
at risk of recurrence and metastatic conversion, two major sources of morbidity for patients having tumor resection with curative intent (3). The perioperative period is well known for activating the body's natural stress response starting with upregulated neuroendocrine signaling, increased release of pro-inflammatory mediators and heightened immunomodulation (4). Additionally, surgical resection of solid tumors leads to increased sympathetic output and invites a pro-inflammatory response to tissue damage which is necessary for tissue repair and healing. This biological response to surgical stress can be hijacked and used for the benefit of any remaining cancer cells to ensure their survival and possibly allow them to migrate. Metastatic disease is the most common cause of death for cancer patients and it can be a source of great financial burden and emotional distress for patients and their families (5).

The biology of cancer cell survival and migration in the perioperative period is frequently studied and is extremely complex (4); hence, much time and effort have been spent to examine two important considerations, the effects of anesthetic techniques and drug choices on the risk of primary recurrence and metastatic conversion for these patients. To date there have been numerous in vitro, in vivo and retrospective studies as well as several prospective randomized controlled trials performed in hopes of addressing these considerations (2, 4, 6-14). In this review, we will report the latest data investigating the role of anesthesia in cancer recurrence and metastatic conversion. It is important to note that to date, there are no official recommendations for best practice in this area. Many studies have suggested some anesthetic agents have the potential to be harmful and increase the risk of recurrence or disease progression while others have been shown to decrease these risks.

A literature search was performed using public databases with the following key words: cancer recurrence, metastatic conversion, general anesthesia, regional anesthesia, total intravenous anesthesia, dexmedetomidine and systemic local anesthetics. Appropriateness for inclusion in the narrative was determined by the authors to include a wide and unbiased range of recent and pertinent studies. Thus in order to examine how anesthesia may affect patient outcomes we will discuss basic tumor biology and some of the potential targets available for modulation. Then we will report and comment on recent studies comparing outcomes for patients undergoing solid tumor resections under general anesthesia $v s$ regional anesthesia followed by outcome data comparing two major types of general anesthesia-volatile anesthesia (e.g., isoflurane, sevoflurane, desflurane) vs propofol based TIVA (Total Intravenous Anesthesia). Lastly, some recent data on the oncogenic effects of various commonly used anesthetic agents will be discussed.

\section{CURRENT STATE}

There have been tremendous advances in the field of cancer biology over the past decade and though our understanding has deepened, there is much that remains a mystery (1). Factors affecting cancer recurrence and metastatic conversion at the time of primary resection are two facets of cancer biology that remain incompletely understood.

There are three basic mechanisms by which recurrence and metastatic conversion occur $(15,16)$. The first is local recurrence where surviving cancer cells may proliferate at the primary site of resection via mechanisms involving pro-inflammatory cytokines, pro-oncogenes and angiogenic factors. Second, cancer cells may transform and acquire the ability to travel to distant sites through either vascular or lymphatic spread due activation and mutation of oncogenes. And third, body cavity seeding during primary tumor resection. The use of intraperitoneal chemotherapy during cytoreductive surgery or primary resection of cancer is one tool aimed at destroying microscopic disease (17-19).

As previously noted, surgical resection of tumors induces an expected state of systemic inflammation and local hypoxia as a result of tissue damage and immunomodulation that may facilitate the conversion of solid tumors into metastatic disease, otherwise known as the epithelial to mesenchymal conversion (20-22). At the same time this pro-inflammatory state exerts a myriad of effects on the body's own cell mediated immune response. There is an intricate interplay between the release of cortisol and catecholamines and the function of immune cells including but not limited to natural killer (NK) cells and CD8+ T cells, both of which are stunted in their antitumor activity. Additionally prooncogenic cell lines, regulatory T cells and type 2 helper T cells are activated and encouraged to proliferate in such a state (4).

It is therefore only logical that anesthesiologists would look to take advantage of the sympatholytic, anti-inflammatory and immunomodulatory effects of anesthetic drugs in an attempt to modify this process and improve patient outcomes. In essence, the ideal anesthetic for cancer patients would:

I. Attenuate sympathetic response while maintaining adequate tissue perfusion to avoid tissue hypoxia

II. Attenuate pro-inflammatory milieu while maintaining an adequate healing response

III. Modulate cytokine release and cellular function to lean toward promoting NK and CD8+ cell activity

IV. Modulate transcription factors and oncogenes to prevent inducible cell survival and migration

Unfortunately, despite promising in vitro and in vivo studies it appears that this process is far more complex than originally thought, likely owed to both the heterogeneous biology of different malignancies and patient populations. Recent prospective randomized clinical trials (RCTs) have shown little promise at elucidating the perfect anti-oncogenic anesthetic, however there are dozens of active multicenter RCTs aimed at shedding light on this topic $(1,23)$.

\section{USE OF GENERAL ANESTHESIA VS REGIONAL ANESTHESIA}

Volatile anesthetics and other hypnotics used to induce and maintain general anesthesia have several anti-inflammatory and 
immunomodulatory effects $(2,24-30)$. Regional anesthetic techniques, ranging from peripheral nerve blocks to neuraxial analgesia, are already employed in many primary tumor resections in order to reduce post-surgical pain and decrease opioid consumption (31-35). From a physiologic point of view, it is logical that one would expect an improvement in recurrence or conversion outcomes, owed to the powerful sympatholytic effects of regional anesthesia in addition to avoidance of the potentially detrimental immunosuppressive effects of volatile anesthetics and opioids. In 2019, one of the largest RCTs to date, evaluated the use of paravertebral nerve blocks (PVB) combined with propofol TIVA in women undergoing primary mastectomy for breast cancer and compared it to volatile anesthesia and conventional opioid analgesia (25). Recurrence occurred in $102(9.8 \%)$ vs 111 (10.4\%) women in the regional anesthesia $v s$ volatile general anesthesia groups, which was found to be statistically significant and passed the study's futility threshold. The study was aborted at that time and no further data was collected. In this study, it was concluded that the use of regional-propofol anesthesia does not impact breast cancer recurrence (25). Although, this study was appropriately powered and the results seem compelling, we must not forget about the extreme heterogeneity of oncologic disease and should apply caution when generalizing studies such as this to other patient populations. More studies are needed in order to definitively recommend regional $v s$ general anesthesia for any given malignancy or patient population. Although with recent advances in surgical technique more and more surgeries can be performed under regional anesthesia (36) it should be noted that nearly all oncological surgeries require general anesthesia in order to be feasible and safe.

\section{TIVA VS VOLATILE GAS ANESTHESIA}

An interesting question remains whether the known effects of volatile anesthetics on immune function are detrimental for cancer recurrence and metastatic conversion. In vitro and in vivo studies have shown that when breast, ovarian and renal cell carcinoma cells are exposed to volatile gases there is increased cytokine release (IL-1/6/8 and TNF), NK and T-cell modulation as well as an increase in growth, angiogenic and migration factors (3, 7, 37-39). However, for other cancer types such as non-small cell lung cancer (NSCLC) exposure to volatile anesthetics has been shown to be suppressive of growth and migration (40). The Cancer and Anesthesia Study (CAN NCT01975064), one of the largest RCTs to study recurrence and survival in breast cancer patients following exposure to general anesthesia, recently published its analysis of first year survival data for 1705 patients with breast cancer (41). These patients were randomized to either a volatile anesthetic $v s$ TIVA with propofol and no difference in survival was observed between the two groups at one year; patients will continue to be followed until 2022. The CAN trial contains two other arms which include patients undergoing primary resection of colorectal cancer which are still in progress. This study points to some important complexities which include the heterogeneity of tumor biology including different cancer types, length of surgery and patient factors such as race and other environmental factors. It was noted in this study that patients of Chinese descent had improved survival rates at one year than other groups (41). To date there has been one RCT that showed propofol decreased local recurrence of breast intraductal carcinoma for patients undergoing primary resection with the goal of breast conservation (42). This study included 2036 women of Asian descent randomized to receive either propofol TIVA and PVB vs volatile anesthesia and PVB. Women who received propofol showed a significant reduction in local recurrence risk; however, there was no difference in risk of metastatic conversion. In short, more data is needed to definitively say whether exposure to one type of anesthetic is beneficial or harmful for the survival of cancer patients.

\section{OPIOIDS}

Due to the world-wide opioid epidemic, the use of opioids in anesthesia has long been under question as there are more and more pharmacologic agents that can be used to manage intraoperative and post-operative pain as well as achieve sympatholysis during general anesthesia. Opioids are powerful immunomodulators which are known to affect innate cell immunity by downregulating NK cell activity and decreasing cytokine production $(31,43)$. This effect is thought to be due to mu-opioid receptor activity as evidenced in one study by improved survival in colorectal and breast cancer patients receiving mu-opioid receptor antagonists, such as naloxone $(44,45)$. Other cell and animal studies have shown that opioids have a direct effect on tumor growth via activation of transcription factors (46). Additionally, opioids have been shown to be pro-angiogenic through activation of VEGF-receptors $(30,45,47)$. For decades it was thought that opioids were largely ubiquitous in their immunomodulatory effects and morphine was used as the prototypical opioid profile; however, with recent data it is becoming clear that different opioids exert different effects on the immune system. For example, morphine and fentanyl have been shown to have similar effects on NK-cell activity and lymphocyte proliferation; however, oxycodone has been shown to have minimal immunosuppressive properties (48). Despite this data, it would be naïve to think that it might be possible to completely eliminate the use of opioids in the treatment of pain in cancer patients as they are the most commonly employed analgesic drugs in the post-operative period (33). Frustratingly, opioid sparing techniques do not seem to affect short term survival as noted in one study that randomized patients to receive remifentanil infusions (47). Thus, the question becomes whether there is a balance of pharmacologic effects between anesthetic and analgesic agents that could be found to improve patient disease free survival.

\section{ALPHA-2 AGONISTS}

Clonidine and dexmedetomidine are powerful $\alpha 2$-adrenoceptor agonists used in general anesthesia and ICU care for their analgesic effects, opioid sparing properties as well as powerful 
sedative and anxiolytic effects. Some studies have found dexmedetomidine to be neuroprotective and an improvement in postoperative cognitive dysfunction through reduction of serum TNF- $\alpha$, IL-6, PI3K and AKT, which would also suggest that dexmedetomidine is anti-inflammatory (49-51). Because of its analgesic properties and excellent performance as a sympatholytic, dexmedetomidine is an alluring choice for use in general anesthesia for cancer patients. Even when compared to clonidine, dexmedetomidine is significantly more efficacious with fewer side effects. There is however, much evidence to suggest the contrary.

There have been numerous in vivo and in vitro studies showing that dexmedetomidine may in fact increase the risk for recurrence by modulating cell survival through activation of HIF- $1 \alpha$ as well as increased secretion of metalloproteinases (MMP) which have been implicated in cell migration and metastatic conversion $(6,9-11,14$, $38,50,52-56)$. The transcription factor HIF-1 $\alpha$ has been shown to confer a survival advantage to cells when exposed to hypoxic conditions, such as when vascular supply is removed during resection $(9,52,54,56)$. Bruzzone et al. first found that $\alpha 2$ adrenoceptors have a positive effect on the proliferation of a mouse mammary tumor cell line in vitro (57). In addition to already discussed effects of HIF- $1 \alpha$, dexmedetomidine induces the proliferation of myeloid-derived suppressor cells associated with significant proangiogenic potential, promoting tumor metastasis through increasing production of VEGF (9). Furthermore, dexmedetomidine upregulates the expression of survivin, MMP -2 , MMP-9, all implicated in metastatic conversion of lung adenocarcinoma (56). A recent retrospective study for patients with NSCLC showed that the use of dexmedetomidine had no benefit on recurrence free survival and a significantly lower overallsurvival for patients who underwent primary surgical resection (56). These effects have been noted in other cancer types such as esophageal, colorectal and hepatocellular carcinoma $(6,7)$.

As with previous hypotheses involving the effects of anesthesia on cancer recurrence and metastatic conversion these data are not practice altering. Quality evidence in support of or against use of dexmedetomidine in clinical practice for cancer patients is lacking. More prospective RCTs are needed to determine whether effects seen in cell and animal studies will pan out. However, with the number of studies suggesting potential harm from dexmedetomidine it is probably prudent to avoid using it if "safer" alternatives are available. There are several RCTs aimed at studying the effects of dexmedetomidine on cancer recurrence. One trial examining the impact of dexmedetomidine on breast cancer recurrence is due to be completed in 2024 (NCT03109990) (23).

\section{LOCAL ANESTHETICS}

Amide local anesthetics, specifically lidocaine, have long been a useful tool in the management of pain during general anesthesia, employed both as systemic intravenous infusions and during neuraxial and peripheral nerve blocks. Lidocaine is a short acting minimally toxic sodium channel blocker that acts to decrease nerve conduction and results in reduction of pain scores in patients receiving intravenous infusions intraoperatively and postoperatively $(35,58-60)$. In addition to its analgesic properties, lidocaine exhibits anti-oncogenic and antiinflammatory effects through various pathways (61-64). Dozens of laboratory studies have been performed to flesh out the biological pathways responsible for lidocaine's observed effects $(12,13,32,65-68)$. Unfortunately, clinical data including retrospective analyses are sparce. To date there has been one study reporting on the observed clinical effects of lidocaine on recurrence of pancreatic cancer, which showed that patients treated with intravenous infusions of lidocaine had better survival rates at 1 and 3 years with no difference in disease-free survival (65). These anti-inflammatory effects have been observed even through alternative methods of local anesthetic administration including intraperitoneal lavage. In one prospective randomized controlled study of patients undergoing ovarian tumor resection, it was observed that patients who received intraperitoneal washings of ropivacaine had a shortened time to chemotherapy administration vs patients in the placebo arm (69). Though the mechanism by which this was achieved is unclear, the authors proposed it could be due to an attenuated inflammatory response, local anesthetic cytotoxicity for microscopic disease in the peritoneum and improved wound healing. While not a direct effect on cancer recurrence, the effect noted in this study could suggest an alternative use for local anesthetics that could hasten a patient's treatment course-several studies have concluded that early chemotherapy administration is associated with improved outcomes although the timeline is still under debate (70).

An upcoming RCT, Volatile Anesthesia and Perioperative Outcomes Related to Cancer trial (VAPOR-C, NCT04316013) set to complete in 2025, will examine the effects of lidocaine in patients with lung or colorectal adenocarcinoma (71). It is important to remember that using intravenous lidocaine as an analgesic is off-label. To date there have been no studies to show that lidocaine infusions are harmful to cancer patients so long as they are employed judiciously and there are no contraindications or conditions that would increase toxicity, such as severe liver disease or low protein states $(32,60,61,71)$. Centers using intravenous lidocaine infusions should have safety protocols and dosing guidelines to avoid harm in patients receiving this treatment (60). Time and care should be applied to training personnel in the recognition of lidocaine toxicity and treatment both intraoperatively and in the post-operative period (61). At our institution, it is common to use intravenous lidocaine infusions for patients that have undergone colorectal surgery, not always to treat oncologic disease, as part of an enhanced recovery after surgery (ERAS) protocol to aid in gut motility and decrease opioid consumption.

\section{CONCLUSION}

Optimization of the care of cancer patients is in constant flux and evolution. The perioperative period has been identified as a unique intersection of intent to treat with potential harm coming to the patient due to that treatment. Anesthesiologists and surgeons are in 


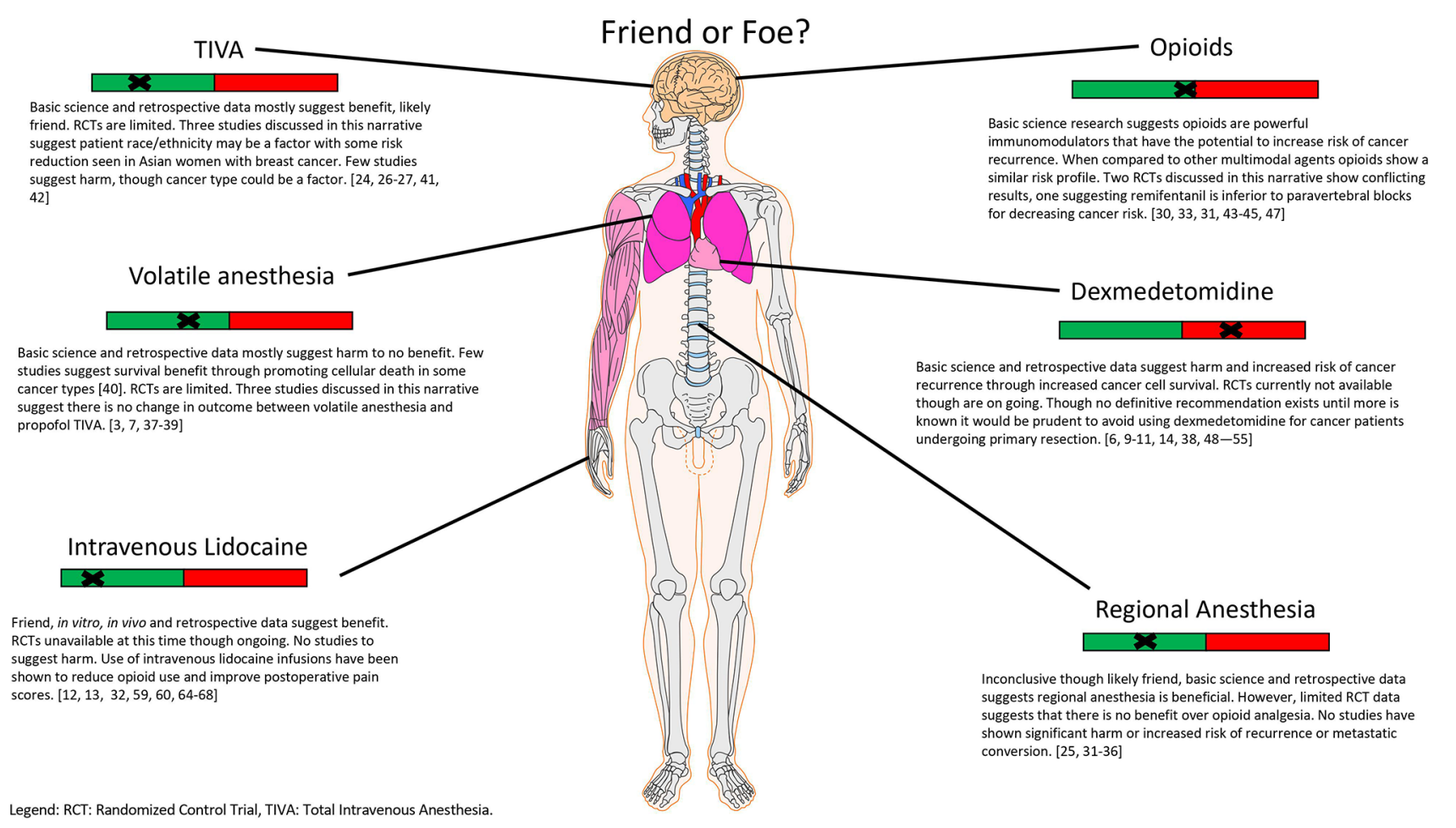

FIGURE 1 | A summary of findings depicted in this narrative review.

the unique position to affect a patient's postoperative course and survival outcome. Specifically, the agents chosen to induce and maintain general anesthesia while surgical intervention is performed have the potential to bring benefit or harm to these patients (Figure 1). In this review, we have briefly discussed cancer cell biology and how recurrence and metastatic conversion may occur as a result of the interplay between circulating tumor cells, cytokines, the HPA axis, the immune system, growth and migration factors and catecholamines as well as the effects of several commonly used hypnotics and analgesics. Despite the numerous studies performed to date, the data currently available is insufficient to form a definitive recommendation for anesthetic choice. Although, from a mechanism point of view, it is tempting to hope that the perfect anesthetic exists for mitigating the risk of cancer recurrence given the vast complexity of oncologic disease and patient genetic heterogeneity it is likely that we may never have an answer. It will likely require genetic phenotyping of patients and their disease to personalize the delivery of anesthesia, while the technology is available it is far from being applied clinically (72-74). Nevertheless, given the immense impact that oncologic disease has worldwide and that it is only projected to continue to worsen this remains an area of high potential for improving the lives of many.

\section{AUTHOR CONTRIBUTIONS}

JM was the first author contributing literature review, writing manuscript as well as design of figure. VJ-T is the senior author contributing expertise in the field of anesthesiology, editing and advising as well as writing of abstract. All authors contributed to the article and approved the submitted version.

\section{REFERENCES}

1. Luo J, Shi Y, Wang X, Zhang R, Chen S, Yu W, et al. A 20-Year Research Trend Analysis of the Influence of Anesthesia on Tumor Prognosis Using Bibliometric Methods. Front Oncol (2021) 11:683232. doi: 10.3389/ fonc.2021.683232

2. Sekandarzad MW, van Zundert AJ, Lirk PB, Doornebal CW, Hollmann MW. Perioperative Anesthesia Care and Tumor Progression. Anesth Analg (2017) 124(5):1697-708. doi: 10.1213/ANE.0000000000001652

3. Forget P, Aguirre JA, Bencic I, Borgeat A, Cama A, Condron C, et al. How Anesthetic, Analgesic and Other Non-Surgical Techniques During Cancer

Surgery Might Affect Postoperative Oncologic Outcomes: A Summary of Current State of Evidence. Cancers (Basel) (2019) 11(5):592. doi: 10.3390/ cancers 11050592

4. Coussens LM, Werb Z. Inflammation and Cancer. Nature (2002) 420 (6917):860-7. doi: 10.1038/nature01322

5. Weingart SN, Nelson J, Koethe B, Yaghi O, Dunning S, Feldman A. Association Between Cancer-Specific Adverse Event Triggers and Mortality: A Validation Study. Cancer Med (2020) 9(12):4447-59. doi: 10.1002/cam4.3033

6. Chen P, Luo X, Dai G, Jiang Y, Luo Y, Peng S. Dexmedetomidine Promotes the Progression of Hepatocellular Carcinoma Through Hepatic Stellate Cell Activation. Exp Mol Med (2020) 52(7):1062-74. doi: 10.1038/s12276-020-0461-6 
7. Deng F, Ouyang M, Wang X, Yao X, Chen Y, Tao T, et al. Differential Role of Intravenous Anesthetics in Colorectal Cancer Progression: Implications for Clinical Application. Oncotarget (2016) 7(47):77087-95. doi: 10.18632/ oncotarget. 12800

8. Nguyen J, Luk K, Vang D, Soto W, Vincent L, Robiner S, et al, Luk K, Vang D, Soto W, Vincent L, Robiner S. Morphine Stimulates Cancer Progression and Mast Cell Activation and Impairs Survival in Transgenic Mice With Breast Cancer. Br J Anaesth (2014) 113(Suppl 1):i4-13. doi: 10.1093/bja/aeu090

9. Su X, Fan Y, Yang L, Huang J, Qiao F, Yu Fang Y, et al. Dexmedetomidine Expands Monocytic Myeloid-Derived Suppressor Cells and Promotes Tumour Metastasis After Lung Cancer Surgery. J Transl Med (2018) 16 (1):347. doi: 10.1186/s12967-018-1727-9

10. Szpunar MJ, Burke KA, Dawes RP, Brown EB, Madden KS. The Antidepressant Desipramine and $\alpha 2$-Adrenergic Receptor Activation Promote Breast Tumor Progression in Association With Altered Collagen Structure. Cancer Prev Res (Phila) (2013) 6(12):1262-72. doi: 10.1158/1940-6207.CAPR-13-0079

11. Tian H, Hou L, Xiong Y, Cheng Q, Huang J. Effect of DexmedetomidineMediated Insulin-Like Growth Factor 2 (IGF2) Signal Pathway on Immune Function and Invasion and Migration of Cancer Cells in Rats With Ovarian Cancer. Med Sci Monit (2019) 25:4655-64. doi: 10.12659/MSM.915503

12. Wall TP, Crowley PD, Buggy DJ. The Effect of Lidocaine and Bosutinib on 4T1 Murine Breast Cancer Cell Behaviour In Vitro. Anticancer Res (2021) 41 (6):2835-40. doi: 10.21873/anticanres.15064

13. Wall TP, Crowley PD, Sherwin A, Foley AG, Buggy DJ. Effects of Lidocaine and Src Inhibition on Metastasis in a Murine Model of Breast Cancer Surgery. Cancers (Basel) (2019) 11(10):1414. doi: 10.3390/cancers11101414

14. Zhang P, He H, Bai Y, Liu W, Huang L. Dexmedetomidine Suppresses the Progression of Esophageal Cancer via miR-143-3p/Epidermal Growth Factor Receptor Pathway Substrate 8 Axis. Anticancer Drugs (2020) 31(7):693-701. doi: 10.1097/CAD.0000000000000934

15. Ganesh K, Massagué J. Targeting Metastatic Cancer. Nat Med (2021) 27 (1):34-44. doi: 10.1038/s41591-020-01195-4

16. Suhail Y, Cain MP, Vanaja K, Kurywchak PA, Levchenko A, Kalluri R, et al. Systems Biology of Cancer Metastasis. Cell Syst (2019) 9(2):109-27. doi: 10.1016/j.cels.2019.07.003

17. Al-Quteimat OM, Al-Badaineh MA. Intraperitoneal Chemotherapy: Rationale, Applications, and Limitations. J Oncol Pharm Pract (2014) 20 (5):369-80. doi: 10.1177/1078155213506244

18. Ghirardi V, Ronsini C, Trozzi R, Di Ilio C, Di Giorgio A, Cianci S. Hyperthermic Intraperitoneal Chemotherapy in Interval Debulking Surgery for Advanced Epithelial Ovarian Cancer: A Single-Center, Real-Life Experience. Cancer (2020) 126(24):5256-62. doi: 10.1002/cncr.33167

19. Revaux A, Carbonnel M, Kanso F, Naoura I, Asmar J, Kadhel P. Hyperthermic Intraperitoneal Chemotherapy in Ovarian Cancer: An Update. Horm Mol Biol Clin Investig (2020) 41(3):2019-0028. doi: 10.1515/hmbci-2019-0028

20. Sakhneny L, Khalifa-Malka L, Landsman L. Pancreas Organogenesis: Approaches to Elucidate the Role of Epithelial-Mesenchymal Interactions. Semin Cell Dev Biol (2019) 92:89-96. doi: 10.1016/j.semcdb.2018.08.012

21. Sannino G, Marchetto A, Kirchner T, Grünewald TGP. Epithelial-ToMesenchymal and Mesenchymal-To-Epithelial Transition in Mesenchymal Tumors: A Paradox in Sarcomas? Cancer Res (2017) 77(17):4556-61. doi: 10.1158/0008-5472.CAN-17-0032

22. Yang J, Weinberg RA. Epithelial-Mesenchymal Transition: At the Crossroads of Development and Tumor Metastasis. Dev Cell (2008) 14(6):818-29. doi: 10.1016/j.devcel.2008.05.009

23. Wall T, Sherwin A, Ma D, Buggy DJ. Influence of Perioperative Anaesthetic and Analgesic Interventions on Oncological Outcomes: A Narrative Review. Br J Anaesth (2019) 123(2):135-50. doi: 10.1016/j.bja.2019.04.062

24. Hong B, Lee S, Kim Y, Lee M, Youn AM, Rhim H. Anesthetics and LongTerm Survival After Cancer Surgery-Total Intravenous Versus Volatile Anesthesia: A Retrospective Study. BMC Anesthesiol (2019) 19(1):233. doi: 10.1186/s12871-019-0914-4

25. Sessler DI, Pei L, Huang Y, Fleischmann E, Marhofer P, Kurz A, et al. Recurrence of Breast Cancer After Regional or General Anaesthesia: A Randomised Controlled Trial. Lancet (2019) 394(10211):1807-15. doi: 10.1016/S0140-6736(19)32313-X
26. Wigmore TJ, Mohammed K, Jhanji S. Long-Term Survival for Patients Undergoing Volatile Versus IV Anesthesia for Cancer Surgery: A Retrospective Analysis. Anesthesiology (2016) 124(1):69-79. doi: 10.1097/ ALN.0000000000000936

27. Yap A, Lopez-Olivo MA, Dubowitz J, Hiller J, Riedel B. Anesthetic Technique and Cancer Outcomes: A Meta-Analysis of Total Intravenous Versus Volatile Anesthesia. Can J Anaesth (2019) 66(5):546-61. doi: 10.1007/s12630-01901330-x

28. Lee EK, Ahn HJ, Zo JI, Kim K, Jung DM, Park JH. Paravertebral Block Does Not Reduce Cancer Recurrence, But Is Related to Higher Overall Survival in Lung Cancer Surgery: A Retrospective Cohort Study. Anesth Analg (2017) 125 (4):1322-8. doi: 10.1213/ANE.0000000000002342

29. Pérez-González O, Cuéllar-Guzmán LF, Soliz J, Cata JP. Impact of Regional Anesthesia on Recurrence, Metastasis, and Immune Response in Breast Cancer Surgery: A Systematic Review of the Literature. Regional Anesthesia Pain Med (2017) 42(6):751-6. doi: 10.1097/AAP.0000000000000662

30. Sen Y, Xiyang H, Yu H. Effect of Thoracic Paraspinal Block-Propofol Intravenous General Anesthesia on VEGF and TGF- $\beta$ in Patients Receiving Radical Resection of Lung Cancer. Med (Baltimore) (2019) 98(47):e18088. doi: 10.1097/MD.0000000000018088

31. Bugada D, Lorini LF, Lavand'homme P. Opioid Free Anesthesia: Evidence for Short and Long-Term Outcome. Minerva Anestesiol (2021) 87(2):230-7. doi: 10.23736/S0375-9393.20.14515-2

32. Chong PH, Yeo ZZ. Parenteral Lidocaine for Complex Cancer Pain in the Home or Inpatient Hospice Setting: A Review and Synthesis of the Evidence. $J$ Palliat Med (2021) 24(8):1154-60. doi: 10.1089/jpm.2020.0622

33. Gabriel RA, Swisher MW, Sztain JF, Furnish TJ, Ilfeld BM, Said ET. State of the Art Opioid-Sparing Strategies for Post-Operative Pain in Adult Surgical Patients. Expert Opin Pharmacother (2019) 20(8):949-61. doi: 10.1080/ 14656566.2019.1583743

34. Kumar K, Kirksey MA, Duong S, Wu CL. A Review of Opioid-Sparing Modalities in Perioperative Pain Management: Methods to Decrease Opioid Use Postoperatively. Anesth Analg (2017) 125(5):1749-60. doi: 10.1213/ ANE.0000000000002497

35. Kutay Yazici K, Menşure K, Büşra A, Süheyla U. The Effect of Perioperative Lidocaine Infusion on Postoperative Pain and Postsurgical Recovery Parameters in Gynecologic Cancer Surgery. Clin J Pain (2021) 37(2):12632. doi: 10.1097/AJP.0000000000000900

36. Mergeay M, Verster A, Van Aken D, Vercauteren M. Regional Versus General Anesthesia for Spine Surgery. A Comprehensive Review. Acta Anaesthesiol Belg (2015) 66(1):1-9.

37. Deng X, Vipani M, Liang G, Gouda D, Wang B, Wei H. Sevoflurane Modulates Breast Cancer Cell Survival via Modulation of Intracellular Calcium Homeostasis. BMC Anesthesiol (2020) 20(1):253. doi: 10.1186/s12871-020-01139-y

38. Liu Y, Sun J, Wu T, Lu X, Du Y, Duan H. Effects of Serum From Breast Cancer Surgery Patients Receiving Perioperative Dexmedetomidine on Breast Cancer Cell Malignancy: A Prospective Randomized Controlled Trial. Cancer Med (2019) 8(18):7603-12. doi: 10.1002/cam4.2654

39. Ciechanowicz S, Zhao H, Chen Q, Cui J, Mi E, Mi E, et al. Differential Effects of Sevoflurane on the Metastatic Potential and Chemosensitivity of NonSmall-Cell Lung Adenocarcinoma and Renal Cell Carcinoma In Vitro. $\mathrm{Br} \mathrm{J}$ Anaesth (2018) 120(2):368-75. doi: 10.1016/j.bja.2017.11.066

40. Wang L, Wang T, Gu J-Q, Su H-B. Volatile Anesthetic Sevoflurane Suppresses Lung Cancer Cells and miRNA Interference in Lung Cancer Cells. Oncol Targets Ther (2018) 11:5689-93. doi: 10.2147/OTT.S171672

41. Enlund M, Enlund A, Berglund A, Bergkvist L. Rationale and Design of the CAN Study: An RCT of Survival After Propofol- or Sevoflurane-Based Anesthesia for Cancer Surgery. Curr Pharm Des (2019) 25(28):3028-33. doi: 10.2174/1381612825666190705184218

42. Zhang J, Chang C-L, Lu C-Y, Chen H-M, Wu S-Y. Paravertebral Block in Regional Anesthesia With Propofol Sedation Reduces Locoregional Recurrence in Patients With Breast Cancer Receiving Breast Conservative Surgery Compared With Volatile Inhalational Without Propofol in General Anesthesia. BioMed Pharmacother (2021) 142:111991. doi: 10.1016/ j.biopha.2021.111991

43. Chen J, Luo F, Lei M, Chen Z. A Study on Cellular Immune Function of Patients Treated With Radical Resection of Pulmonary Carcinoma With Two Different Methods of Anesthesia and Analgesia. J buon (2017) 22(6):1416-21. 
44. Bimonte S, Barbieri A, Cascella M, Rea D, Palma G, Del Vecchio V, et al. The Effects of Naloxone on Human Breast Cancer Progression: In Vitro and In Vivo Studies on MDA.MB231 Cells. Onco Targets Ther (2018) 11:185-91. doi: 10.2147/OTT.S145780

45. Ma M, Wang X, Liu N, Shan F, Feng Y. Low-Dose Naltrexone Inhibits Colorectal Cancer Progression and Promotes Apoptosis by Increasing M1Type Macrophages and Activating the Bax/Bcl-2/Caspase-3/PARP Pathway. Int Immunopharmacol (2020) 83:106388. doi: 10.1016/j.intimp.2020.106388

46. Wu Q, Chen X, Wang J, Sun P, Weng M, Chen W. Nalmefene Attenuates Malignant Potential in Colorectal Cancer Cell via Inhibition of Opioid Receptor. Acta Biochim Biophys Sin (Shanghai) (2018) 50(2):156-63. doi: 10.1093/abbs/gmx131

47. Yan T, Zhang GH, Wang BN, Sun L, Hui Zheng H. Effects of Propofol/ Remifentanil-Based Total Intravenous Anesthesia Versus Sevoflurane-Based Inhalational Anesthesia on the Release of VEGF-C and TGF- $\beta$ and Prognosis After Breast Cancer Surgery: A Prospective, Randomized and Controlled Study. BMC Anesthesiol (2018) 18(1):131. doi: 10.1186/s12871-018-0588-3

48. Franchi S, Moschetti G, Amodeo G, Sacerdote P. Do All Opioid Drugs Share the Same Immunomodulatory Properties? A Review From Animal and Human Studies. Front Immunol (2019) 10(2914). doi: 10.3389/fimmu.2019.02914

49. Zhang J, Liu G, Zhang F, Fang H, Zhang D, Liu S, et al. Analysis of Postoperative Cognitive Dysfunction and Influencing Factors of Dexmedetomidine Anesthesia in Elderly Patients With Colorectal Cancer. Oncol Lett (2019) 18(3):3058-64. doi: 10.3892/ol.2019.10611

50. Liu M, Yi Y, Zhao M. Effect of Dexmedetomidine Anesthesia on Perioperative Levels of TNF- $\alpha$ and IL-6 in Patients With Ovarian Cancer. Oncol Lett (2019) 17(6):5517-22. doi: 10.3892/ol.2019.10247

51. Huyan T, Hu X, Peng H, Zhu Z, Li Q, Zhang W. Perioperative Dexmedetomidine Reduces Delirium in Elderly Patients After Lung Cancer Surgery. Psychiatr Danub (2019) 31(1):95-101. doi: 10.31219/osf.io/e9hfw

52. Zhu L, Zhang Y, Zhang Z, Ding X, Gong C, Qian Y. Activation of PI3K/Akt/ HIF-1 $\alpha$ Signaling is Involved in Lung Protection of Dexmedetomidine in Patients Undergoing Video-Assisted Thoracoscopic Surgery: A Pilot Study. Drug Des Devel Ther (2020) 14:5155-66. doi: 10.2147/DDDT.S276005

53. Yi XL, Wang JT, Chu CQ, Li YX, Yin JH, Liu SL. Cardiocerebral Protective Effects of Dexmedetomidine as Anesthetic in Colorectal Cancer Surgery. Eur Rev Med Pharmacol Sci (2018) 22(11):3570-6. doi: 10.26355/eurrev_201806_15183

54. Chen HY, Li GH, Tan GC, Liang H, Lai XH, Huang Q, et al. Dexmedetomidine Enhances Hypoxia-Induced Cancer Cell Progression. Exp Ther Med (2019) 18(6):4820-8. doi: 10.3892/etm.2019.8136

55. Xia M, Ji NN, Duan ML, Tong JH, Xu JG, Zhang YM, et al. Dexmedetomidine Regulate the Malignancy of Breast Cancer Cells by Activating $\alpha 2$ Adrenoceptor/ERK Signaling Pathway. Eur Rev Med Pharmacol Sci (2016) 20(16):3500-6.

56. Cata JP, Singh V, Lee BM, Villarreal J, Mehran JR, Yu J, et al. Intraoperative Use of Dexmedetomidine is Associated With Decreased Overall Survival After Lung Cancer Surgery. J Anaesthesiol Clin Pharmacol (2017) 33(3):317-23. doi: 10.4103/joacp.JOACP_299_16

57. Lavon H, Matzner P, Benbenishty A, Sorski L, Rossene E, Haldar R, et al. Dexmedetomidine Promotes Metastasis in Rodent Models of Breast, Lung, and Colon Cancers. Br J Anaesth (2018) 120(1):188-96. doi: 10.1016/j.bja.2017.11.004

58. Weibel S, Jelting Y, Pace NL, Helf A, Eberhart LHJ, Hahnenkamp K, et al. Continuous Intravenous Perioperative Lidocaine Infusion for Postoperative Pain and Recovery in Adults. Cochrane Database Syst Rev (2018) 6(6): Cd009642. doi: 10.1002/14651858.CD009642.pub3

59. Masic D, Liang E, Long C, Sterk EJ, Barbas B, Rech MA. Intravenous Lidocaine for Acute Pain: A Systematic Review. Pharmacotherapy (2018) 38 (12):1250-9. doi: 10.1002/phar.2189

60. Khan JS, Hodgson N, Choi S, Reid S, Paul JE, Look Hong NJ, et al. Perioperative Pregabalin and Intraoperative Lidocaine Infusion to Reduce Persistent Neuropathic Pain After Breast Cancer Surgery: A Multicenter, Factorial, Randomized, Controlled Pilot Trial. J Pain (2019) 20(8):980-93. doi: 10.1016/j.jpain.2019.02.010

61. Lee JT, Sanderson CR, Xuan W, Agar M. Lidocaine for Cancer Pain in Adults: A Systematic Review and Meta-Analysis. J Palliat Med (2019) 22(3):326-34. doi: $10.1089 /$ jpm.2018.0257

62. Hermanns H, Hollmann MW, Stevens MF, Lirk P, Brandenburger T, Piegeler $\mathrm{T}$, et al. Molecular Mechanisms of Action of Systemic Lidocaine in Acute and
Chronic Pain: A Narrative Review. Br J Anaesth (2019) 123(3):335-49. doi: 10.1016/j.bja.2019.06.014

63. Galoș EV, Tat T-F, Popa R, Efrimescu C-I, Finnerty D, Buggy DJ, et al. Neutrophil Extracellular Trapping and Angiogenesis Biomarkers After Intravenous or Inhalation Anaesthesia With or Without Intravenous Lidocaine for Breast Cancer Surgery: A Prospective, Randomised Trial. Br J Anaesth (2020) 125(5):712-21. doi: 10.1016/j.bja.2020.05.003

64. van Haren F, van den Heuvel S, Radema S, van Erp N, van den Bersselaar L, Vissers K, et al. Intravenous Lidocaine Affects Oxaliplatin Pharmacokinetics in Simultaneous Infusion. J Oncol Pharm Pract (2020) 26(8):1850-6. doi: $10.1177 / 1078155220905011$

65. Zhang H, Yang L, Zhu X, Zhu M, Sun Z, Cata JP, et al. Association Between Intraoperative Intravenous Lidocaine Infusion and Survival in Patients Undergoing Pancreatectomy for Pancreatic Cancer: A Retrospective Study. Br J Anaesth (2020) 125(2):141-8. doi: 10.1016/j.bja.2020.03.034

66. Ji W, Zhang X, Sun G, Wang X, Liu J, Bian J, et al. Effect of Perioperative Intravenous Lidocaine on Postoperative Outcomes in Patients Undergoing Resection of Colorectal Cancer: A Protocol for Systematic Review and MetaAnalysis. BMJ Open (2021) 11(8):e048803. doi: 10.1136/bmjopen-2021-048803

67. Freeman J, Crowley PD, Foley AG, Gallagher HC, Iwasaki M, Ma D, et al. Effect of Perioperative Lidocaine, Propofol and Steroids on Pulmonary Metastasis in a Murine Model of Breast Cancer Surgery. Cancers (Basel) (2019) 11(5):613. doi: 10.3390/cancers 11050613

68. Hou YH, Shi WC, Cai S, Liu H, Zheng Z, Qi FW, et al. Effect of Intravenous Lidocaine on Serum Interleukin-17 After Video-Assisted Thoracic Surgery for Non-Small-Cell Lung Cancer: A Randomized, Double-Blind, PlaceboControlled Trial. Drug Des Devel Ther (2021) 15:3379-90. doi: 10.2147/ DDDT.S316804

69. Hayden JM, Oras J, Block L, Thörn S-E, Palmqvist C, Salehi S, et al. Intraperitoneal Ropivacaine Reduces Time Interval to Initiation of Chemotherapy After Surgery for Advanced Ovarian Cancer: Randomised Controlled Double-Blind Pilot Study. Br J Anaesth (2020) 124(5):562-70. doi: 10.1016/j.bja.2020.01.026

70. Hofstetter G, Concin N, Braicu I, Chekerov R, Sehouli J, Cadron I, et al. The Time Interval From Surgery to Start of Chemotherapy Significantly Impacts Prognosis in Patients With Advanced Serous Ovarian Carcinoma - Analysis of Patient Data in the Prospective OVCAD Study. Gynecologic Oncol (2013) 131(1):15-20. doi: 10.1016/j.ygyno.2013.07.086

71. Wall TP, Buggy DJ. Perioperative Intravenous Lidocaine and Metastatic Cancer Recurrence - A Narrative Review. Front Oncol (2021) 11:688896. doi: $10.3389 /$ fonc. 2021.688896

72. Bach-Rojecky L, Vađunec D, Lozić M, Žunić K, Špoljar GG, Čutura T, et al. Challenges in Anesthesia Personalization: Resolving the Pharmacogenomic Puzzle. Per Med (2019) 16(6):511-25. doi: 10.2217/pme-2019-0056

73. Xie S, Ma W, Guo Q, Liu J, Li W, McLeod HL, et al. The Pharmacogenetics of Medications Used in General Anesthesia. Pharmacogenomics (2018) 19 (3):285-98. doi: 10.2217/pgs-2017-0168

74. McKinstry-Wu AR, Wasilczuk AZ, Harrison BA, Bedell VM, Sridharan MJ, Breig JJ, et al. Analysis of Stochastic Fluctuations in Responsiveness is a Critical Step Toward Personalized Anesthesia. Elife (2019) 8:e50143. doi: 10.7554/eLife.50143

Conflict of Interest: The authors declare that the research was conducted in the absence of any commercial or financial relationships that could be construed as a potential conflict of interest.

Publisher's Note: All claims expressed in this article are solely those of the authors and do not necessarily represent those of their affiliated organizations, or those of the publisher, the editors and the reviewers. Any product that may be evaluated in this article, or claim that may be made by its manufacturer, is not guaranteed or endorsed by the publisher.

Copyright (๑) 2021 Montejano and Jevtovic-Todorovic. This is an open-access article distributed under the terms of the Creative Commons Attribution License (CC BY). The use, distribution or reproduction in other forums is permitted, provided the original author(s) and the copyright owner(s) are credited and that the original publication in this journal is cited, in accordance with accepted academic practice. No use, distribution or reproduction is permitted which does not comply with these terms. 